\title{
Determination and comparison miR135a in the serum between women with GDM, non- pregnant type 2 diabetes, healthy pregnant and control group
}

\author{
Yousef Khazaei Monfared (1) \\ Fatemeh Ghadimi (1) \\ Farshad Foroughi (2) \\ Maryam Honardoost (3) \\ Sima Hashemipour (4) \\ Fatemeh Sefidi (5) \\ Mohamad Reza Sarookhani (6)
}

(1) Social Determinants of Health Research Center, Qazvin University of Medical Sciences, Qazvin, Iran.

(2) Department Of Immunology, School Of Medicine, Qazvin University Of Medical Science, Qazvin, Iran

(3) Endocrine Research Center, Institute of Endocrinology and Metabolism, Iran University of Medical Sciences, Tehran, Iran

(4) Metabolic Diseases Research Center, Qazvin University of Medical Sciences, Qazvin, Iran

(5) Employment in Dental faculty, Qazvin University of Medical Sciences, Qazvin,Iran

(6) Cellular And Molecular Research Center, Qazvin University Of Medical Sciences, Qazvin, Iran

\section{Corresponding Address:}

Mohamad Reza Sarookhani

Cellular And Molecular Research Center, Qazvin University Of Medical Sciences,

P.O.Box: $934197-5981$

Qazvin, Iran

Email: sarokhani2002@yahoo.com

\section{Abstract \\ Objectives: Diabetes is one of the most important endocrine diseases caused by complex reactions between genetic and environmental factors. Recent studies have shown that microRNAs play an impor- tant role in the production, inhibition, and secretion of insulin. Identifying the relationship between key miRNAs that control the genes involved in the patho- genesis of diabetes is clinically important because it provides a way to identify preventive methods or treatments. In the present study, the expression of miR135a in serum samples between women with Gestational diabetes mellitus (GDM), non-pregnant type 2 diabetes, and healthy pregnant women were compared with the control group.}

Materials and methods: This study was a case-control study and non-random sampling method was used. The present study was conducted among four groups (healthy non-pregnant women (control), non-pregnant Diabetes type 2, GDM, and healthy pregnant). After serum separation, expression of miR-135a was measured using QRT-PCR technique and the results were analyzed by Stata and SPSS21 software.
Results: The results show that the mean expression of miR-135a gene in control group was $0.9 \pm 0.06$, control of pregnancy was $1 \pm 0.1$, GDM group was $1.7 \pm 0.3$ and non-pregnant diabetic type 2 group was $6 \pm 6 / 3$. The results of analysis of variance showed that the mean difference of miR-135 gene expression was significant higher in the non- pregnant type 2 diabetes than GDM group ( $F=2776.3$, $P<0.001)$.

Conclusion: The widespread role of miRNAs as post-transplantation gene regulators in gestational diabetes mellitus suggests that miR135a may act as a potential indicator of the prevention, treatment, and management of gestational diabetes .

Key words: miR135a, non- pregnant type 2 diabetes, gestational diabetes mellitus, QRT-PCR

Please cite this article as: Sarookhani M.R. et al.

Determination and comparison miR135a in the serum between women with GDM, non- pregnant type 2 diabetes, healthy pregnant and control group 2018;16(2):193-197. World Family Medicine.

DOI: 10.5742/MEWFM.2018.93267 